\section{Messabweichung, quadratischer Mittelwert}

C. Vidal ${ }^{1}$ und W.-R. Külpmann ${ }^{2}$

${ }^{1}$ Landeskriminalamt Niedersachsen, Dezernat 53 „Chemie“, Hannover, Deutschland

${ }^{2}$ Hannover, Deutschland

Englischer Begriff mean square of measurement deviation

Definition Maß für die Streuung der Messwerte einer Probe um den Zielwert.

Beschreibung Der quadratische Mittelwert der Messabweichung $\left(\Delta^{2}\right)$ wird wie folgt berechnet:

$$
\Delta^{2}=\frac{1}{\mathrm{n}} \times \sum_{\mathrm{i}=1}^{\mathrm{n}}\left(\mathrm{x}_{\mathrm{i}}-\mathrm{x}_{0}\right)^{2}
$$

$\mathrm{n}=$ Anzahl der zur Berechnung herangezogenen Einzelergebnisse

$\mathrm{x}_{0}=$ Zielwert

$\mathrm{x}_{\mathrm{i}}=$ Messergebnis der Einzelmessung
Statt $\Delta^{2}$ wird in der Regel $\Delta$, d. h. die Quadratwurzel des quadratischen Mittelwerts der Messabweichung verwendet (engl.: root mean square of measurement deviation).

Zwischen dem quadratischen Mittelwert der Messabweichung $\left(\Delta^{2}\right)$ einerseits und der systematischen Messabweichung ( $\triangleright$ Messabweichung, systematische) und der empirischen - Standardabweichung einer Stichprobe von Messergebnissen einer Probe besteht rechnerisch folgender Zusammenhang:

$$
\Delta^{2}=\frac{\mathrm{n}-1}{\mathrm{n}} \times \mathrm{s}^{2}+\delta^{2}
$$

$$
\begin{aligned}
& \delta=\text { Bias } \\
& \mathrm{s}=\text { empirische Standardabweichung }
\end{aligned}
$$

\section{Literatur}

Macdonald R (2006) Quality assessment of quantitative analytical results in laboratory medicine by root mean square of measurement deviation. J Lab Med 30:111-117

Richtlinie der Bundesärztekammer zur Qualitätssicherung laboratoriumsmedizinischer Untersuchungen (2014) Dtsch Arztebl 111: A1583-A1618 